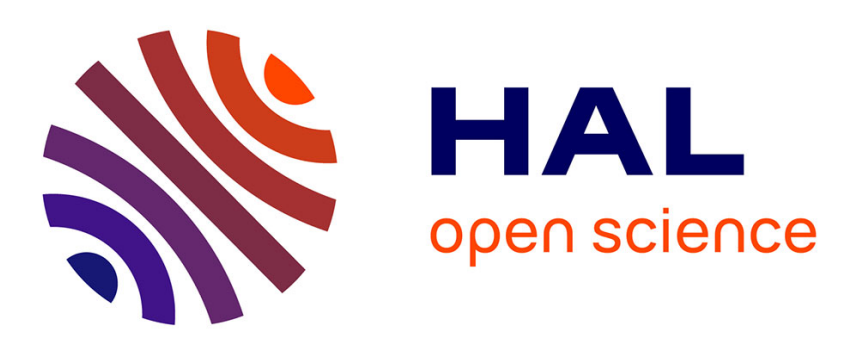

\title{
Electronic Structure, Electron-Phonon Coupling, and Charge Transport in Crystalline Rubrene Under Mechanical Strain
}

\author{
Michael Ruggiero, Sergio Ciuchi, Simone Fratini, Gabriele D'avino
}

\section{To cite this version:}

Michael Ruggiero, Sergio Ciuchi, Simone Fratini, Gabriele D'avino. Electronic Structure, ElectronPhonon Coupling, and Charge Transport in Crystalline Rubrene Under Mechanical Strain. Journal of Physical Chemistry C, 2019, 123 (26), pp.15897-15907. 10.1021/acs.jpcc.9b01902 . hal-03011519

\author{
HAL Id: hal-03011519 \\ https://hal.science/hal-03011519
}

Submitted on 3 Dec 2020

HAL is a multi-disciplinary open access archive for the deposit and dissemination of scientific research documents, whether they are published or not. The documents may come from teaching and research institutions in France or abroad, or from public or private research centers.
L'archive ouverte pluridisciplinaire HAL, est destinée au dépôt et à la diffusion de documents scientifiques de niveau recherche, publiés ou non, émanant des établissements d'enseignement et de recherche français ou étrangers, des laboratoires publics ou privés. 


\section{Electronic Structure, Electron-Phonon Coupling}

and Charge Transport in Crystalline Rubrene

\section{Under Mechanical Strain}

Michael T. Ruggiero, ${ }^{\dagger}$ Sergio Ciuchi, ${ }^{\ddagger}$ Simone Fratini, ${ }^{\llbracket}$ and Gabriele D’Avino, ${ }^{*}$

$\dagger$ Department of Chemistry, University of Vermont, 82 University Place, Burlington, VT 05405, United States of America

$\ddagger$ Department of Physical and Chemical Sciences, University of L’Aquila, Via Vetoio, L'Aquila, Italy I-67100

ๆInstitut Néel, CNRS and Université Grenoble Alpes, BP 166, F-38042 Grenoble, France

E-mail: gabriele.davino@neel.cnrs.fr 


\begin{abstract}
Motivated by the potential for application of organic semiconductors in flexible electronics, we present a theoretical study aiming at elucidating the interplay between mechanical strain and electronic, vibrational and charge transport properties of the prototypical high-mobility molecular semiconductor rubrene. Our study considers several factors that can play a role in the electro-mechanical response of a soft, van-der-Waals bonded, molecular crystal, such as intermolecular charge transfer integrals, lattice dynamics and electron phonon coupling. We find that compressive strain leads to an increase in magnitude of charge transfer integrals but also of the energetic disorder hampering the mobility. Charge transport simulations, based on the transient localization framework and fed with first-principles inputs, reveal a remarkably different response to strain applied along different crystal axes, in line with most recent experiments. The critical interplay between energetic disorder of intrinsic and extrinsic nature on the mobility-strain relationship is also discussed. The theoretical approach proposed in this work paves the way for the systematic study of the electro-mechanical response of different classes of high-mobility molecular semiconductors.
\end{abstract}

\title{
Introduction
}

Mechanical flexibility is one of the key assets of organic semiconductors that makes these materials promising candidates for functional elements in the next generation of flexible, foldable, and wearable electronic devices. Indeed, applications of organic electronics in flexible microelectronic circuits and display technologies have been demonstrated in recent years. ${ }^{1-5}$ For these applications a certain stability of electrical properties of the semiconductor under mechanical deformation (strain) is required, while, for other uses, such as actuators of pressure sensors, a pronounced electro-mechanical responsiveness is in demand. Thus, it is critical to achieve a complete understanding of the interplay between mechanical and electrical properties in order to effectively utilize these materials for advanced applications. 
Additionally, because these materials are inherently bound by weak intermolecular forces, such an understanding can arguably contribute to shed light on the fundamentals of the mechanism of charge transport in soft molecular semiconductors.

The focus of the present manuscript is on single crystals ${ }^{6-9}$ that, in principle, represent ideal systems for the investigation of the intrinsic relationship between charge transport and mechanical strain, i.e. the response in the absence of disorder or crystalline defects that dominate the electrical and electro-mechanical properties of amorphous or polycrystalline organic semiconductors. Recent studies on single crystals agree in reporting increases in the charge mobility $\mu$ with compressive strain $\epsilon,{ }^{6-9}$ the latter defined as $\epsilon=\left(l-l_{0}\right) / l_{0}$, being $l$ and $l_{0}$ the sample length in the presence and in the absence of a mechanical deformation along a given direction. Substantial differences have been, however, reported on quantitative aspects. By taking the dimensionless strain factor $g=\frac{d\left(\mu-\mu^{0}\right) / \mu^{0}}{d \epsilon}\left(\mu^{0}\right.$ is the mobility in the absence of strain) as a measure of the electro-mechanical response of a given system, the values reported in the literature for the best characterized rubrene crystal range from $g \sim 6^{9}$ (i.e. $6 \%$ mobility variation at $1 \%$ strain) to $g \sim 200 .{ }^{6}$ Most of the data are relative to the longitudinal response, i.e. when mobility is measured along the direction of the applied strain, usually the high- $\mu$ crystal axis $b{ }^{6,8}$ The full tensor response, including transverse components, has been recently measured by Matta et al.,${ }^{9}$ who reported larger off-diagonal responses of magnitude comparable to diagonal ones. Similar results have been also disclosed in a preprint by Batlogg and collaborators. ${ }^{10}$

The large variability in the reported experimental estimates of $g$ might be attributed to extrinsic effects of different nature (e.g. chemical impurities, traps, gate dielectric, etc.) specific to materials batches or to different measurement setups. Moreover, most of the literature data were obtained from two-terminal measurements, ${ }^{6,8,9}$ which may be affected by contact resistance artifacts related to strain-dependent injection barriers, as suggested by scanning Kelvin probe microscopy measurements. ${ }^{11}$ Considering the above mentioned factors hampering the measurements' reproducibility, theoretical calculations represent a 
unique resource for the investigation of the intrinsic electro-mechanical response of molecular semiconductors.

The present work builds on the nowadays well-acknowledged fact that charge transport in soft molecular solids is strongly hampered by lattice vibrations. ${ }^{12-16}$ This has been successfully described within the transient localization theory, which provided a comprehensive account of a complex experimental scenario. ${ }^{17,18}$ Within this framework, mechanical strain may affect the charge transport in several different ways. In first instance, (i) the lattice compression may increase the overlap between adjacent molecules, resulting in an increment of the magnitude of intermolecular charge transfer integrals. This intuitive scenario has been confirmed by band structure calculations ${ }^{7,11}$ and transfer integrals statistics over molecular dynamics trajectories. ${ }^{9}$ On the other hand, (ii) the change in transfer integrals may affect the isotropy of the electronic band structure and with that its resilience to dynamic energetic disorder. ${ }^{18}$ For instance, rubrene is a rather anisotropic, quasi one-dimensional material, characterized by transfer integrals of much larger magnitude along the high-mobility crystal axis $b$ than along all other directions. ${ }^{19,20}$

Strain may also impact the overall magnitude of the energetic disorder due to thermal lattice vibrations, firstly (iii) by affecting intermolecular non-covalent forces, for instance leading to the stiffening of the lattice upon compression as suggested in Ref. 7. (iv) In second instance, lattice deformations could influence the electron-phonon (e-ph) coupling, i.e. the sensitivity of the electronic structure to lattice vibrations. Considering all the above mentioned effects, which can in principle cooperate or counteract each other, a careful comprehensive theoretical analysis is needed to determine how these factors will combine in determining the ultimate dependence of mobility on strain, and to identify the leading ones, if any.

In the present paper, we present a density functional theory (DFT) study on the effect of mechanical strain on the electronic structure, lattice dynamics and e-ph coupling in the paradigmatic and best-characterized molecular semiconductor rubrene. ${ }^{21}$ The results of our 
first-principles calculations are then used to assess the impact of strain on the hole mobility within the framework of the transient localization framework. ${ }^{18}$ Compressive strain is found to lead to a stiffening of the crystal lattice and to an increase of both the magnitude of intermolecular charge transfer integrals and energetic disorder. Charge transport simulations reveal a much stronger mobility response to strain applied along the crystal axis $c$ with respect to $b$, and suggest that extrinsic disorder, present in real devices, can significantly impact the electro-mechanical response. 


\section{Methods}

The effects of mechanical strain on the structure, electronic properties and lattice dynamics of the prototypical molecular semiconductor rubrene has been investigated by means of solidstate first-principles calculations and rationalized within the framework of a tight-binding model with coupling to lattice vibrations. We have considered the most common orthorombic phase $^{22}$ of the rubrene with four molecules in the conventional cell, which is characterized by high-mobility bc planes sketched in Figure 1.

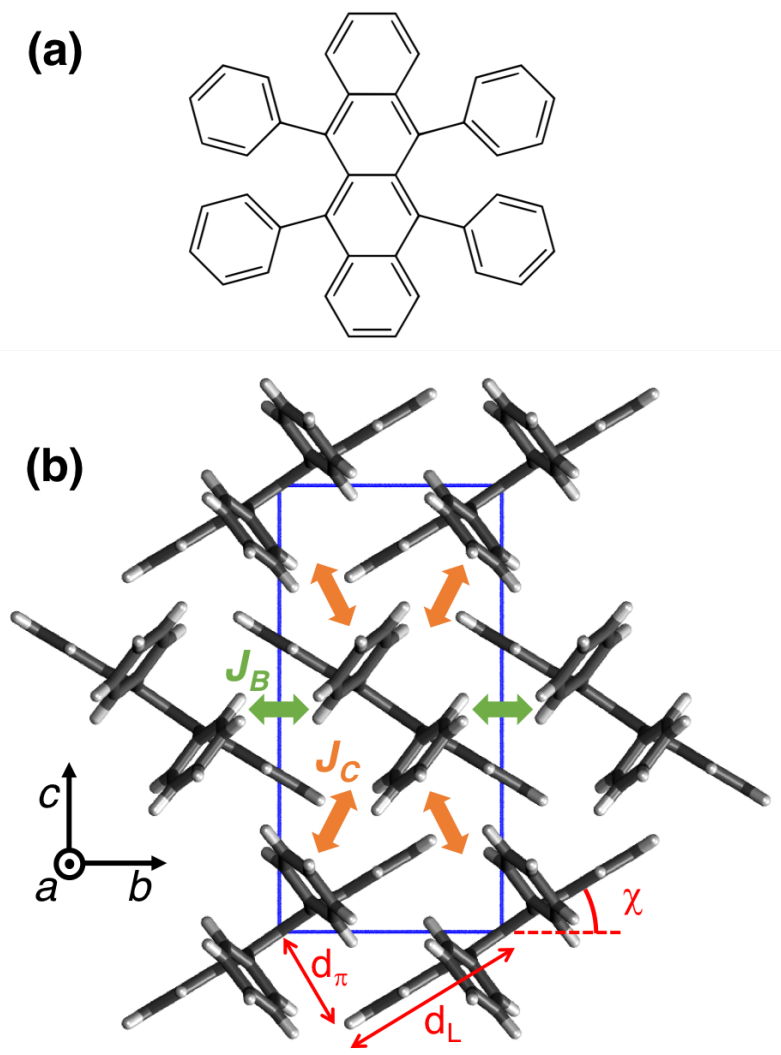

Figure 1: Illustration of (a) the rubrene molecular structure and of (b) the crystal packing in the high-mobility $b c$ planes. Transfer integrals between symmetry-nonequivalent molecular pairs, $J_{B}$ and $J_{C}$ are shown in green and orange, respectively. The geometrical parameters determining the molecular packing are shown in red.

The reference framework for the electronic structure of a molecular semiconductor is a 
tight binding model with Hamiltonian

$$
H=\sum_{m} \varepsilon_{m} a_{m}^{\dagger} a_{m}+\sum_{m n}^{\prime} J_{m n} a_{m}^{\dagger} a_{n},
$$

where $\epsilon_{m}$ is the energy of the molecular site $m, J_{m n}$ is the charge transfer integral between neighboring sites $m$ and $n$, and $a_{m}^{\dagger}\left(a_{m}\right)$ is the creation (annihilation) operator of a charge on site $m$. In the following, we will consider a two-dimensional system corresponding to the rubrene $b c$ planes (see Figure 1) where the relevant site orbitals are the highest occupied molecular orbital (HOMO), as pertinent to hole transport.

The linear coupling to lattice vibrations is introduced within a real-space framework, similar to previous studies. ${ }^{23-27}$ To such a purpose, we consider the set of normal-mode Cartesian coordinates $q_{i}$ with frequencies $\omega_{i}$ obtained from periodic DFT calculations (see below), where $i$ is a composite index labelling the wave vector and the branch of the phonon mode. For local (Holstein) couplings we consider the modulation of site energies:

$$
\varepsilon=\varepsilon^{0}+\sum_{i} \beta_{i}^{H} q_{i}
$$

where $\epsilon^{0}$ is the energy of is the site energy at equilibrium and $\beta_{i}^{H}$ is the Holstein coupling constant. Nonlocal (Peierls) couplings is introduced through the modulation of transfer integrals

$$
J_{x}=J_{x}^{0}+\sum_{i} \beta_{i}^{x} q_{i}
$$

where $J_{x}^{0}$ the transfer integral at equilibrium and $\beta_{i}^{x}$ is the Peierls coupling constant, relative to the pair of molecules $x=\mathrm{B}, \mathrm{C}$, as sketched in Figure 1b. The strength of the e-ph coupling is quantified by the mode-specific relaxation energies (also called polaron binding energy)

$$
\lambda_{i}^{x}=\frac{\left(\beta_{i}^{x}\right)^{2}}{2 m_{i} \omega_{i}},
$$

for local $(x=\mathrm{H})$ and nonlocal $(x=\mathrm{B}, \mathrm{C})$ coupling, being $m_{i}$ the effective mass of mode $i$. 
The thermal population of the lattice modes results in dynamic energetic disorder, i.e. a spread of site energies and transfer integrals due to thermal lattice fluctuations. For linear coupling to quantum harmonic modes, the distribution of the parameters entering Hamiltonian 1 is Gaussian with variance

$$
\sigma_{x}^{2}=\frac{\hbar}{2 N} \sum_{i} \frac{\left(\beta_{i}^{x}\right)^{2}}{m_{i} \omega_{i}} \operatorname{coth}\left(\frac{\hbar \omega_{i}}{2 k_{B} T}\right)
$$

for local (spread in $\epsilon, x=\mathrm{H}$ ) and nonlocal (spread in $J_{x}, x=\mathrm{B}, \mathrm{C}$ ) coupling, where $T$ is the temperature, $\hbar$ the reduced Planck constant, $k_{B}$ the Boltzmann constant and $N$ corresponds to the number of points taken into account in the sampling of the phonon spectrum over the Brillouin zone.

\section{Crystal structure and lattice dynamics under strain}

Our analysis relies on periodic DFT calculations based on the Perdew-Becke-Ernzerhof (PBE) functional ${ }^{28}$ with Grimme's D3-BJ pairwise van der Waals corrections. ${ }^{29,30}$ This approach has been recently shown to be among the most accurate methods to describe the subtle interplay between covalent and weak interactions in molecular crystals. ${ }^{31,32}$ All-electron fully-periodic DFT calculations have been performed with the CRYSTAL17 code. ${ }^{33}$

We have first optimized the cell parameters and atomic coordinates in the absence of strain, checking the convergence of the results with respect to the computational parameters such as the basis set, up to $6-311 \mathrm{G}(2 \mathrm{~d}, 2 \mathrm{p})$, and the Brillouin zone sampling, up to $8 \times 8 \times 8)$. The results reported in Table 1 demonstrate the good agreement between our calculations and experimental structures, and show that a satisfactory description of forces is achieved employing the $6-31 \mathrm{G}(\mathrm{d})$ basis set and a $2 \times 2 \times 2$ sampling of the Brillouin zone, yielding an average error in unit cell lengths of $-1.45 \%$. These settings were then used in the following solid-state calculations.

Uniaxial strain along a given crystallographic direction has been induced by appropriately 
Table 1: Comparison of the rubrene orthorombic structures from X-ray diffraction and DFT calculations. A good agreement is obtained between DFToptimized and experimental cell parameters. The two optimizations, differing for basis set and Brillouin zone sampling, yield very similar results for the cell parameters and the atomic positions, with a root mean square deviation and maximum absolute deviation of fractional coordinates of $4.710^{-3}$ and $8.910^{-3}$, respectively.

\begin{tabular}{llll}
\hline structure & $a(\AA)$ & $b(\AA)$ & $c(\AA)$ \\
\hline exp. $[293 \mathrm{~K}]^{22}$ & 26.860 & 7.193 & 14.433 \\
exp. $[100 \mathrm{~K}]^{22}$ & 26.789 & 7.170 & 14.211 \\
calc. $[6-311 \mathrm{G}(2 \mathrm{~d}, 2 \mathrm{p}), 8 \times 8 \times 8]$ & 26.507 & 7.122 & 14.012 \\
calc. $[6-31 \mathrm{G}(\mathrm{d}), 2 \times 2 \times 2]$ & 26.295 & 7.131 & 13.933 \\
\hline
\end{tabular}

scaling the corresponding crystal axis. The other cell parameters were left unchanged, while the atomic coordinates were fully relaxed for each value of the strain. Harmonic lattice dynamics calculations were then performed on the unstrained and strained structures to determine the normal modes and the corresponding frequencies according to established procedures based on the numerical evaluation of the Hessian matrix. ${ }^{34-36}$

Available inelastic neutron scattering data for deuterated naphthalene ${ }^{37}$ and theoretical calculations ${ }^{38-40}$ have shown that the low-frequency (below $200 \mathrm{~cm}^{-1}$ ) phonon modes of molecular crystals feature a pronounced wave vector dependence. The effect of such phonon dispersion on e-ph coupling and on energetic disorder has been addressed only very recently. ${ }^{40-43}$ Brédas and coworkers reported the convergence of the cumulative nonlocal energetic disorder upon systematically increasing the Brillouin zone sampling, from considering the zone center only ( $\Gamma$ point) to full phonon dispersion, for the prototypical herringbone systems such as naphthalene and pentacene. ${ }^{41,42}$ These results showed that almost the totality of the cumulative energetic disorder ( $84 \%$ for pentacene, $93 \%$ for naphthalene) could be recovered by considering a sampling of the Brillouin zone that includes only two points, i.e. $\Gamma$ and the zone-boundary point corresponding to the real-space direction joining neighboring molecules that are equivalent by translational symmetry. In the case of the rubrene crystal, the real-space direction at stake is the crystal axis $b$ (see Figure 1b) and the corresponding zone-boundary point is $Y=(0,0.5,0)$. The importance of accounting for the modes at 
this zone-boundary point lies in the fact that they modulate the out-of-phase vibration of neighboring molecules that are missed at $\Gamma$, including acoustic branches. Considering the high computational cost of all-electron DFT phonon calculations on supercells, which are required to account for phonon dispersion, we opt for a minimal sampling of the Brillouin zone that includes the points $\Gamma$ and $Y(1 \times 2 \times 1$ supercell $)$.

\section{Electron-phonon model parameters}

Local and nonlocal coupling constants, $\beta_{i}^{H}=\frac{\partial \varepsilon}{\partial q_{i}}$ and $\beta_{i}^{x}=\frac{\partial J_{x}}{\partial q_{i}}(x=\mathrm{B}, \mathrm{C})$, have been evaluated with two-point central finite differences. $\epsilon$ and $J_{x}$, were computed from gas-phase DFT calculations on single molecules and molecular dimers at the crystal equilibrium geometry, deformed according to the atomic displacements of each normal mode. This procedure has been iterated for the equilibrium structure and the normal modes previously computed for each value of the applied strain. DFT calculations for molecules and dimers employed the hybrid PBE0 functional ${ }^{44}$ as implemented in the ORCA v4.0 code. ${ }^{45}$ We employed the def2-TZVP basis set for calculations of transfer integrals at equilibrium geometries $J_{x}^{0}$. The smaller def2-SVP basis, in conjunction with the RIJCOSX approximation, ${ }^{46}$ accurately describes variations of HOMO energies and transfer integrals as a function of atomic displacements and was hence used in the calculation of e-ph couplings. Charge transfer integrals $J_{x}$ between the relevant molecular orbitals were computed with the projection dimer approach. ${ }^{47}$

\section{Transient localization mobility simulations}

Charge transport calculations were performed within the transient localization framework in the relaxation time approximation (RTA). ${ }^{13,48}$ The mobility can be obtained from the calculation of the square transient localization length $L_{\tau}^{2}$ on a time-scale $\tau$ dictated by the 
time scale associated to molecular motion as described in details in ${ }^{18}$

$$
\mu=\frac{e}{k_{B} T} \frac{L_{\tau}^{2}}{2 \tau}
$$

The key quantity $L_{\tau}^{2}$ is obtained using exact diagonalization method on finite-size clusters as described in a recent publication. ${ }^{49}$ This approach is computationally cheaper and allows for a better control over size effects with respect to time propagation techniques employed in previous works. ${ }^{18}$ The code is freely available for download at GitHub. ${ }^{50}$

The square transient localization length is computed in terms of eigenstates $|n\rangle$ and eigenvalues $E_{n}$ of Hamiltonian Eq. (1) for a given realization of the disorder as

$$
L_{\tau}^{2}=\frac{2}{Z} \sum_{n, m} \frac{|\langle n|\hat{\mathcal{J}}| m\rangle|^{2} e^{-\beta E_{n}}}{\left(E_{m}-E_{n}\right)^{2}+1 / \tau^{2}}
$$

where $\hat{\mathcal{J}}$ is the current operator, $Z=\sum_{n} e^{-\beta E_{n}}$ is the partition function and $\beta=1 / k_{B} T$. The actual $L_{\tau}^{2}$ to be used in Equation 6 is obtained upon averaging over disorder realizations. ${ }^{51}$ Note that for hole carriers, the thermal weighting factor must be adapted by changing the sign in the exponent.

We have performed exact diagonalizations on a system including up to $48 \times 48$ unit cells (having 2 molecules per cell) with periodic boundary conditions as to ensure the convergence of the calculated mobility values at 300 K. 50 different realizations of disorder have been considered for the averaged mobility.

\section{Results}

\section{Crystal structure and transfer integrals}

We start our analysis from the effect of strain on the crystal structure. Since the four molecules in the orthorombic cell of rubrene are equivalent by symmetry, the molecular 
packing can be characterized in terms of three geometrical parameters illustrated in Figure 1b. These parameters are the distance between the centers of mass of two slipped stacked molecules along the crystal axis $b$, and precisely its components normal to the tetracene cores $\left(d_{\pi}\right)$ and along the core long axis $d_{L}$, and the angle formed by the core long axis and the crystal axis $b(\chi)$. The strain dependence of these structural parameters obtained from DFT optimizations, shown in Figure 2, reflects a non trivial interplay of steric repulsion and cohesive forces in a crystal of anisotropic molecules. Firstly, we notice that the three geometrical parameters present a linear dependence on strain up to values of strain of $\pm 3 \%$. Such a large strain values are unrealistic, since it has been reported that cracks develop in rubrene for $|\epsilon>0.4| \%{ }^{8}$ The lattice compression has the effect of reducing the distance between the $\pi$ planes, $d_{\pi}$, with comparable magnitude for strain along the $b$ and $c$ axes (see Figure 2a,d). Different trends are instead observed for the other two parameters depending on the direction of the applied strain. Compression along $b$ leads to a decrease of the long-axis slip $d_{L}$, while the tilt angle $\chi$ is almost insensitive to the strain (see Figure $2 \mathrm{~b}, \mathrm{c}$ ). For strain along $c, d_{L}$ increases upon compression with a simultaneous decrease of $\chi$ (see Figure 2e,f).

The change in the equilibrium crystal structure with strain directly impacts the electronic properties, such as the intermolecular charge transfer integrals. Due to the nodal structure of molecular $\pi$-conjugated orbitals, transfer integrals are notably highly sensitive to the relative molecular positions, as extensively studied for several molecular semiconductors, including rubrene. ${ }^{20}$ The hole transfer integrals between slipped $\left(J_{B}\right)$ and herringbone $\left(J_{C}\right)$ rubrene molecules (see Figure 1b), shown in Figure 3, both present a linear increase in magnitude upon compression. The values we computed for the unstrained system, $J_{B}=112.4 \mathrm{meV}$ and $J_{C}=-25.9 \mathrm{meV}$, are in line with previous calculations at a comparable level of theory. ${ }^{20,52,53}$ The trends in $J_{B}$ (see Figure $3 \mathrm{a}, \mathrm{c}$ ) seem to be governed by the decrease in the distance between the $\pi$-conjugated molecular planes $d_{\pi}$ (see Figure 2a,d) that enhances the overlap between the rubrene HOMOs. This is supported by the fact that the variations in $J_{B}$ are approximately 1.5 times larger for strain along $c$ with respect to strain along $b$ (see 

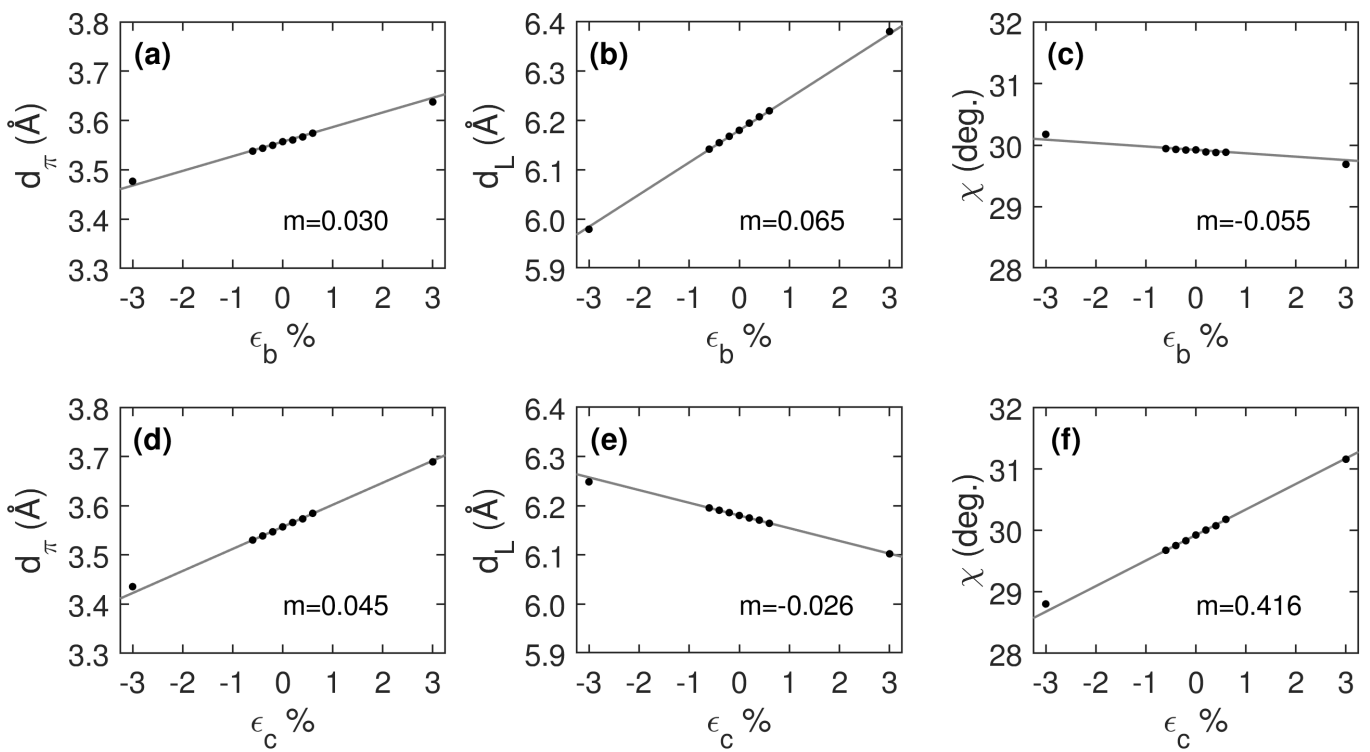

Figure 2: Strain dependence of the molecular organization in the rubrene crystal as defined in Figure $1 \mathrm{~b}$ and in the main text. The grey lines are linear regressions (slope $m$ annotated in each panel) computed with data points for $|\epsilon|<0.6 \%$.

Figure 3a,c), and the same ratio is observed for the variations of $d_{\pi}$ for strain along the same axes see Figure 2a,d). On the other hand, it has been reported that the hole transfer integrals between slipped molecules in the rubrene crystal are weakly sensitive to the longaxis displacements, ${ }^{20}$ making the variation of $d_{L}$ (see Figure 2b,e) less relevant for $J_{B}$. The relative variations of $J_{C}$ with strain along $b$ and $c$, shown in Figure $3 \mathrm{~b}, \mathrm{~d}$, are comparable to those obtained for $J_{B}$, although their interpretation is less straightforward.

In general, we observe an increase in magnitude of transfer integral upon compression that semi-quantitatively confirms very recent DFT results by Gali et al., ${ }^{54}$ and that is in line with the intuitive expectation based on the enhancement of intermolecular overlaps with the shrinking of the lattice. We emphasize the fact that strain has a negligible effect on the anisotropy in the charge transfer integrals as quantified by the ratio $J_{B} / J_{C}=-4.33$. In fact, this ratio varies by less than $1 \%$ for realistic strain values of $|\epsilon|<0.6 \%$ and by less than $5 \%$ for $\epsilon= \pm 3 \%$. We can therefore exclude that the lattice compression or expansion would lead to a change in the isotropy of the electronic structure and hence its sensitivity to energetic 
disorder. $^{18}$
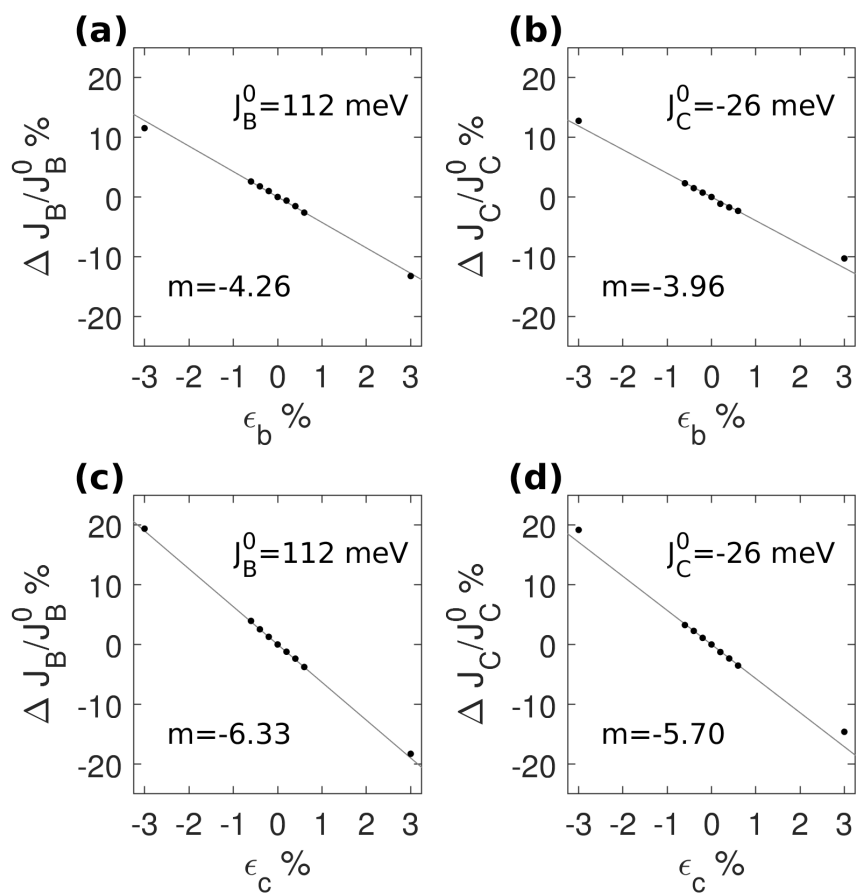

Figure 3: Relative variations of intermolecular charge transfer integrals with strain along the $b$ (top panels) and $c$ (bottom panels axis. An increase in magnitude of transfer integrals is observed upon compression. The grey lines are linear regressions (slope $m$ annotated in each panel) computed with data points for $|\epsilon|<0.6 \%$. The values of $J_{B}$ and $J_{C}$ in the absence of strain are also annotated.

\section{Lattice dynamics under strain}

Having established how the structure and the transfer integrals depend on the strain applied along different crystallographic directions, we now turn our attention to the effect of strain on the lattice dynamics. Figure 4 shows the ratio between the harmonic frequency of the strained and unstrained crystal of each normal mode. The correspondence between the normal modes of the strained and unstrained system has been established on the basis of the scalar product between the vectors of the atomic displacements of each mode, i.e. picking the eigenvectors of the dynamical matrix with the largest projection. Projection values above 0.8 were found for all the modes, in most cases above 0.9 , testifying a safe one-to-one 
correspondence of the modes upon application of a mechanical deformation. As can be seen in Figure 4, lattice compression tends to increase the frequency in the low wavenumbers region, while the opposite is observed upon expansion. Only a few modes seem not to follow the general trend of a lattice stiffening upon compression. This behavior follows the intuitive argument that, in a compressed lattice, molecules experience more the steeper repulsive intermolecular potential. The effect of strain is instead minimal for modes above $200 \mathrm{~cm}^{-1}$. This is somehow expected given that the higher frequency modes are predominantly intramolecular in nature, which is also confirmed by their dispersionless character. ${ }^{43}$ The effects of strain on phonon frequencies are found to be qualitatively similar for strain applied along $b$ and $c$ axis.
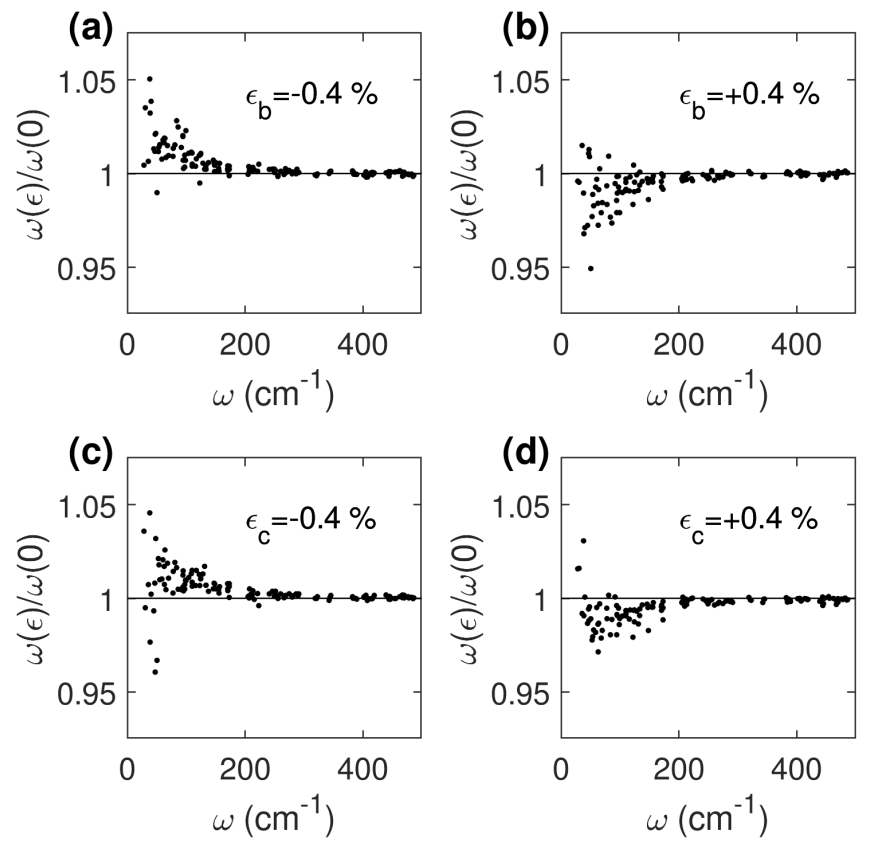

Figure 4: Plots of the ratio between the phonon modes frequencies of the strained (strain values annotated in each panel) and unstrained rubrene crystal. Low-frequency modes show a tendency for hardening upon compression, while the opposite occurs with lattice expansion. Modes above $200 \mathrm{~cm}^{-1}$ are almost insensitive to strain.

The hardening of the low-frequency modes, i.e. those that are mostly responsible for dynamic energetic disorder hampering charge transport in organic semiconductors, implies a reduction in the amplitude of the corresponding atomic oscillations. A reduced amplitude 
upon compression suggests a possible reduction in the dynamic disorder and hence a possible beneficial effect on the mobility. A similar argument has been proposed by Kubo et al., who reported the stiffening of the intermolecular potentials upon compression in a 7 -molecule cluster of $\mathrm{C}_{10}$-DNBDT-NW. ${ }^{7}$ While emphasizing the greater rigour of our approach, which relies on the lattice dynamics obtained from periodic solid-state calculations, we remark that energetic disorder depends both on vibrational amplitudes and on the e-ph coupling. The latter may also be affected by strain, as we investigate next.

\section{Electron-phonon coupling}

The modulation of molecular site energies and intermolecular transfer integrals due to lowfrequency lattice modes of the rubrene crystals has been calculated at the DFT level, within the established framework for linear local and nonlocal e-ph coupling, ${ }^{23-25}$ as detailed in the Methods section. Results in Figure 5a-c report the mode-specific relaxation energies $\left(\lambda^{x}\right.$, gray vertical bars) for local and nonlocal couplings in the unstrained system. In order to better appreciate the frequency distribution of the coupling strength in the case of many modes, possibly very close in frequency or even degenerate, we also consider the spectral function

$$
\Lambda^{x}(\omega)=\sum_{i} \lambda_{i}^{x} \delta\left(\omega-\omega_{i}\right)
$$

where $\delta$ is the Dirac delta function (replaced by Gaussians of $5 \mathrm{~cm}^{-1}$ standard deviation for visualization purpose) and $x=H, B, C$ for local (Holstein) and nonlocal coupling relative to transfer integrals $J_{B}$ and $J_{C}$ (see Figure 1), respectively. The black line in Figure 5a-c reports $\Lambda^{x}(\omega)$ as obtained with our two-point sampling of the Brillouin $(\Gamma+Y$, see Methods section), while the green line is for $\Gamma$-point phonons only. We remark that zone-boundary $Y$ point modes lift the symmetry-equivalence between the molecules in the unit cell. Relaxation energies have been computed by explicitly considering the symmetry-nonequivalent molecules and dimers, and then averaged in the displayed results. 
Panels d-i of Figure 5 show the cumulant values of the relation energies,

$$
\Lambda_{\Sigma}^{x}(\omega)=\sum_{i}^{\omega_{i}<\omega} \lambda_{i}^{x}=\int_{0}^{\omega} \Lambda^{x}\left(\omega^{\prime}\right) d \omega^{\prime}
$$

for strain applied along the crystal axis $b$ (panels d-f) and $c$ (panels g-i). $\Lambda_{\Sigma}^{x}(\omega)$ gives a direct quantification of the overall strength of the coupling due to all modes below a given frequency threshold $\omega$. For low-frequency modes, the classical limit $\left(\hbar \omega \ll k_{B} T\right)$ applies to Equation 5 and $\Lambda_{\Sigma}^{x}$ is proportional to the variance of site energies $(x=\mathrm{H})$ or charge transfer integrals $(x=\mathrm{B}, \mathrm{C})$.

We start the presentation of our results from local e-ph couplings of the unstrained system in Figure 5a. Our results for the coupling to $\Gamma$-point modes are consistent with literature data. ${ }^{25,53}$ Interestingly, the comparison of zone-center results with those obtained upon including zone-boundary modes (c.f. black and green line in Figure 5a,d) reveals that the neglect of phonon dispersion results in an order of magnitude underestimation of the magnitude of the Holstein coupling. The cumulative relaxation energy at $\omega=400 \mathrm{~cm}^{-1}$ is 3.2 and $39.3 \mathrm{meV}$ upon neglecting and accounting for phonons dispersion, respectively.

To the best of our knowledge, the present work reports the first investigation of local e-ph coupling in a molecular crystal beyond the $\Gamma$ point, since all previous studies of e-ph coupling accounting for phonon dispersion focused only on the nonlocal contribution. ${ }^{40-43}$ Literature results show that a few low-frequency modes of the isolated molecule characterized by a remarkable coupling, ${ }^{20,25}$ and involving the bending of the phenyl groups, seem to be silent in the crystal, according to $\Gamma$-point e-ph calculations. ${ }^{25,53}$ Our results hence suggest that phonon dispersion is essential to reconcile the large discrepancy in the low-frequency Holstein spectral weight between the isolated molecule and the crystal when only zone-center modes are accounted for. ${ }^{25}$ An illustration of the zone-boundary modes with the strongest coupling is provided in Figure 6a. We observe that the two modes at 58.6 and $266.0 \mathrm{~cm}^{-1}$ involve a substantial motion of the phenyl groups, similar to a low-frequency and strongly 

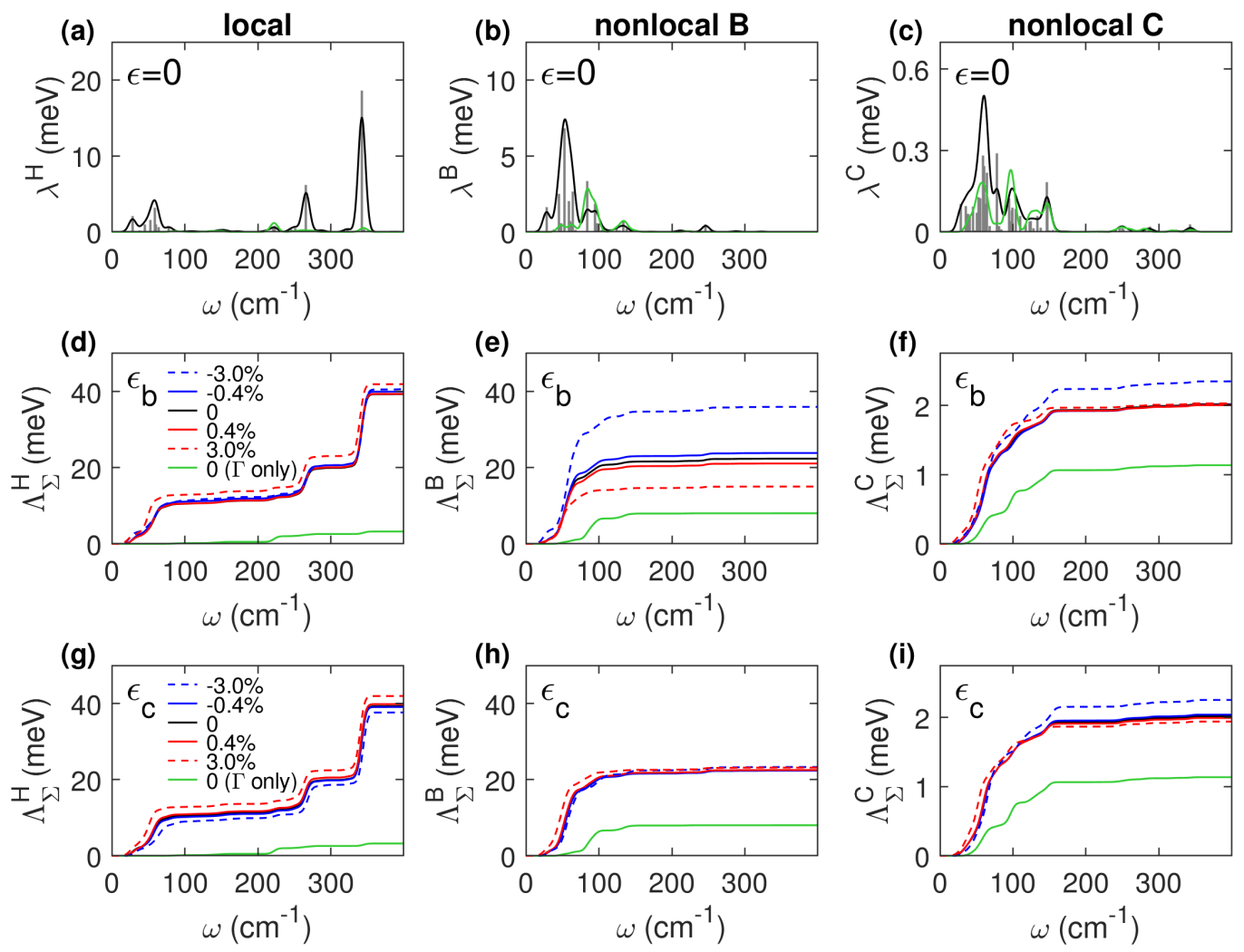

Figure 5: Local (left panels) and nonlocal (center and right panels) e-ph couplings for the strained rubrene crystal. (a-c) Relaxation energies (gray bars) and the corresponding spectral density (black line, arbitrary units) in the unstrained system. (d-f) Cumulative spectral functions for different values of strain (see legend) applied along the $b$ axis. (g-i) Same as panels d-f for strain along $c$. Green lines shows $\Lambda^{x}$ and $\Lambda_{\Sigma}^{x}$ calculated with $\Gamma$-point modes only. The delta function in Eq. 8 has been replaced by a Gaussian with $5 \mathrm{~cm}^{-1}$ standard deviation. 
(a) $\quad Y 58.6 \mathrm{~cm}^{-1}$

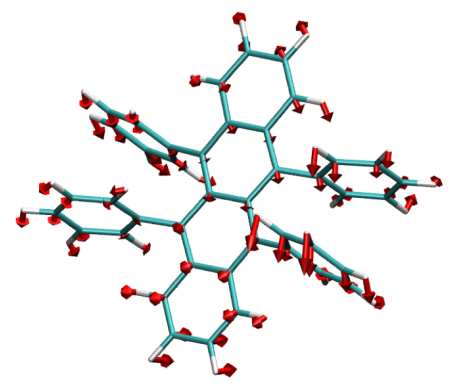

Y $266.0 \mathrm{~cm}^{-1}$

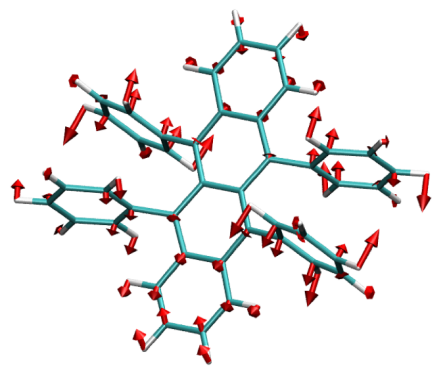

Y $342.5 \mathrm{~cm}^{-1}$

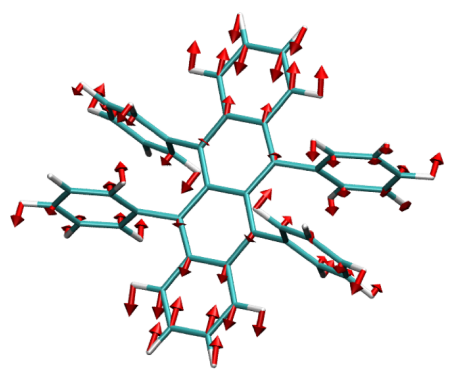

$\Gamma 83.8 \mathrm{~cm}^{-1}$ (b)

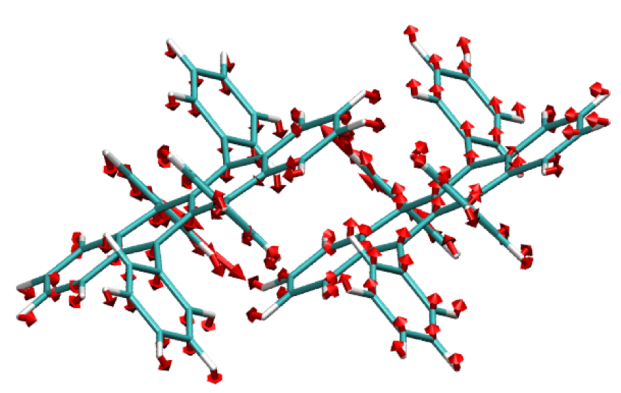

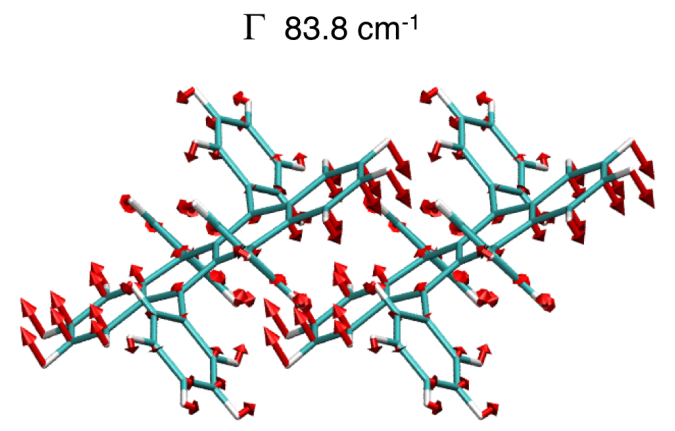

Figure 6: Illustration of the atomic displacements (arrows) for the vibrational modes with strongest (a) local and nonlocal (b) e-ph coupling (see Figure 5). The wavevector (Brillouin zone point $\Gamma$ or $Y=(0,0.5,0)$ and the frequency of each modes are annotated. 
coupled mode of the isolated molecule. ${ }^{20}$

Local e-ph coupling is rather insensitive to strain, as can be evinced from data in Figure $5 \mathrm{~d}, \mathrm{~g}$. The curves of $\Lambda_{\Sigma}^{H}(\omega)$ for experimentally attainable strain values of $\pm 0.4 \%$ are practically superimposed to that of the unstrained system, and modest variations are obtained also for the unrealistically high strain of $\pm 3 \%$.

Coming to nonlocal couplings, our results for the unstrained system in Figure 5b,c are in line with those by Xie et al., ${ }^{40}$ with a much stronger coupling for $J_{B}$ with respect to $J_{C}$, with most of the spectral weight ascribable to modes below $100 \mathrm{~cm}^{-1}$. The sketch of the modes with the strongest nonlocal couplings in Figure 6b confirms a substantial mixing between intra-molecular and intermolecular degrees of freedom. ${ }^{25,40}$ The $\Gamma$-point vibration at $83.8 \mathrm{~cm}^{-1}$ closely resembles a mode reported in Ref. 25 at similar frequency $\left(87 \mathrm{~cm}^{-1}\right)$ and corresponding to quasi-rigid molecule oscillations about the crystal axis $a$. We report, also for nonlocal couplings, a substantial increase in the cumulative relaxation energy when accounting for modes beyond the Brillouin zone center (see Figure 5b,c), similar to recent literature results for rubrene ${ }^{40}$ and other systems. ${ }^{41-43}$

The effect of strain on nonlocal electron-phonon coupling can be appreciated from the plots of the cumulative relaxation energies in Figure 5e-f,h-i. The most noticeable result is the increase of $\Lambda_{\Sigma}^{B}$ in Figure 5e upon lattice compression and its decrease upon expansion, signaling an enhancement in the sensitivity of the largest transfer integrals $J_{B}$ to the lattice motion upon shrinking the crystal. Although the extent of this variation might seem small on the figure scale, we remark that the relative variations of $\Lambda_{\Sigma}^{B}$ at $400 \mathrm{~cm}^{-1}(\sim 10 \%$ for $1 \%$ strain) are larger in magnitude than the relative variation in $J_{B}^{0}$ (c.f. Figure 3 ). The variations of $\Lambda_{\Sigma}^{B}$ with strain are monotonic in $\epsilon_{b}$ up to $\pm 3 \%$, yet the trend is not linear. The effect of transverse strain on $\Lambda_{\Sigma}^{B}$ (see Figure $5 \mathrm{~h}$ ) or of strain along both directions on $\Lambda_{\Sigma}^{C}$ (Figure 5f,i) is much less pronounced, unless unrealistically large lattice deformations of $\pm 3 \%$ are applied. 


\section{Transient localization mobility calculations}

On the basis of the present DFT analysis of the rubrene crystal, the effect of compressive strain can be summarized as follows: (i) increase in the magnitude of hole transfer integrals that, however, does not affect the anisotropy of the electronic structure, i.e. rubrene remains a quasi-1D system with a $J_{B} / J_{C}$ ratio essentially unchanged; (ii) stiffening of the crystal lattice, which implies the shrinking of the thermal vibrational amplitude of low-frequency modes; (iii) enhancement of the nonlocal e-ph coupling only for strain applied along crystal axis $b$, as opposed to a strain-independent local coupling. The net effect of these three factors on the hole transport properties is hardly predictable, since (i) is expected to barely affect the intrinsic mobility, ${ }^{18}$ (ii) is expected to enhance it, while the opposite is expected from (iii). We have hence performed mobility calculations as a function of strain within the framework of the relaxation time approximation (RTA, see Methods for details). ${ }^{13,48,49}$

Charge transport calculations based on the tight binding model (Equation 1) take as input parameters transfer integrals and dynamic energetic disorder from DFT calculations summarized in Table 2. It is well acknowledged that charge transport is hampered dominantly by low-frequency modes. We hence report in Table 2 the standard deviations computed, according to Equation 5, including the effect of all modes with frequencies below $400 \mathrm{~cm}^{-1}$. The actual choice of this threshold is irrelevant for the nonlocal coupling, since strongly coupled modes are all well-below the cutoff frequency (see Figure 5b,c). The same plausible threshold is somewhat arbitrary for the local e-ph, where coupled modes can be found at higher frequencies. Local coupling is, however, insensitive to strain and a possible increase in $\sigma_{H}$ would sum up to the effect of extrinsic disorder discussed below.

Additional parameters in the simulations are the effective phonon frequency $\omega_{0}$, and the amount of extrinsic disorder, if present. The effective phonon frequency $\omega_{0}$ sets the timescale over which lattice vibrations reshuffle the energy landscape experienced by charge carriers, conferring a diffusive character to transport. ${ }^{13}$ This parameter enters in Equation 7 through the timescale $\tau=1 / \omega_{0}$. Since many modes contribute to the dynamic disorder, 
Table 2: Strain dependence of transfer integrals and local and nonlocal energetic disorder at $300 \mathrm{~K}$, i.e. the quantities entering RTA charge transport simulations whose results are shown in Figure 7 . The last column shows the ratio between $J=\sqrt{J_{B}^{2}+2 J_{C}^{2}}$, setting the energy scale of the electronic bandwidth, and $\sigma=$ $\sqrt{\sigma_{B}^{2}+2 \sigma_{C}^{2}+\sigma_{H}^{2} / 4}$, i.e. the total intrinsic disorder (see text). Energies are in meV.

\begin{tabular}{lrrrrrr}
\hline strain \% & $J_{B}$ & $J_{C}$ & $\sigma_{H}$ & $\sigma_{B}$ & $\sigma_{C}$ & $J / \sigma$ \\
\hline$\epsilon=0$ & 112.4 & -25.9 & 48.1 & 34.2 & 10.3 & 2.670 \\
\hline$\epsilon_{b}=-3.0$ & 125.3 & -29.2 & 48.9 & 43.4 & 11.1 & 2.526 \\
$\epsilon_{b}=-0.4$ & 114.4 & -26.3 & 48.4 & 35.3 & 10.3 & 2.661 \\
$\epsilon_{b}=0.4$ & 110.6 & -25.5 & 48.1 & 33.2 & 10.3 & 2.674 \\
$\epsilon_{b}=3.0$ & 97.5 & -23.3 & 49.4 & 28.0 & 10.3 & 2.568 \\
\hline$\epsilon_{c}=-3.0$ & 134.1 & -30.9 & 47.2 & 35.0 & 10.9 & 3.138 \\
$\epsilon_{c}=-0.4$ & 115.2 & -26.5 & 47.9 & 34.2 & 10.4 & 2.737 \\
$\epsilon_{c}=0.4$ & 109.7 & -25.3 & 48.3 & 34.3 & 10.2 & 2.601 \\
$\epsilon_{c}=3.0$ & 91.8 & -22.1 & 49.5 & 34.8 & 10.1 & 2.154 \\
\hline
\end{tabular}

we take the harmonic mean of the vibrational frequencies weighted by the sum of local and nonlocal relaxation energies, which yields $\omega_{0}=67.5 \mathrm{~cm}^{-1}$. We further notice that this value corresponds to the frequency of the most strongly Peierls-coupled modes (c.f. Figure 5b). We have checked that the varying $\omega_{0}$ by up to a factor 2 has a negligible impact on the relative mobility variations with strain and has a small effect on the absolute values of $\mu .{ }^{18}$ Extrinsic disorder cannot be quantified from our calculations for a defect-free crystal and is in general hardly accessible in atomistic simulations. We hence adopt a phenomenological description of extrinsic disorder, that we model with an additional Gaussian diagonal disorder term with strain-independent standard deviation $\Delta$, which is taken as an adjustable parameter.

We start by observing that the absolute value of the mobility we compute for the unstrained rubrene crystal when accounting for intrinsic energetic disorder only $(\Delta=0)$, $\mu_{b}^{0}=24.4 \mathrm{~cm}^{2} \mathrm{~V}^{-1} \mathrm{~s}^{-1}$ (see also Table 3), nicely compares with the top-performing samples measured by Ren in one of the most reliable set of experiments on single-crystal air-gap transistors. ${ }^{55}$

The dependence of mobility on strain is shown in Figure 7 for different values of strainindependent extrinsic disorder up to $\Delta / J(\epsilon=0)=0.8 \approx 95 \mathrm{meV}$, where $J=\sqrt{J_{B}^{2}+2 J_{C}^{2}}$, 
sets the energy scale of the electronic bandwidth for a given strain value. The relative variations of the isotropic mobility $\mu=\left(\mu_{b}+\mu_{c}\right) / 2$ for strain applied along the $b$ and $c$ axes are shown in the top and bottom panels, respectively. Results implicitly account for the trivial effect of the lattice deformation, which affects the unit of length and hence the calculated mobility. We notice that in the absence of extrinsic disorder $(\Delta=0)$, our simulations predict opposite effects for strain applied along different directions, i.e. the mobility increases with $\epsilon_{b}$ and decreases with $\epsilon_{c}$. While the latter trend is in line with experimental observations, showing an increase of $\mu$ upon compression, ${ }^{6,8,9}$ the effect of strain along the crystal axis $b$ is unexpected.

It is however well known that real organic crystals and field effect transistors (FETs) are always affected by a certain amount of extrinsic disorder, due to crystallographic defects, impurities, and gate dielectric. The introduction of extrinsic disorder degrades the calculated mobility (see $\mu^{0}$ values in Table 3 ) and, most importantly, qualitatively alters the response to strain applied along $b$. Indeed, compression along $b$ leads to an increase in $\mu$ upon compression for $\Delta / J(0)>0.5$, which reconciles present calculations with experiments regarding the qualitative trend of mobility enhancement with compression. For strain along $c$, extrinsic disorder further enhances the response of the system to strain.

Table 3: Mobility of the unstrained system $\left(\mu^{0}\right.$ components in $\left.\mathbf{c m}^{2} \mathbf{V}^{-1} \mathbf{s}^{-1}\right)$ and strain factor tensor $\left(g_{i j}=\frac{d\left(\Delta \mu_{i} / \mu_{i}^{0}\right)}{d \epsilon_{j}}\right)$ calculated as a function of the extrinsic disorder $\Delta$. Extrinsic disorder reduces the absolute values of the mobility and enhances the electro-mechanical response of the system, favoring mobility enhancements upon compression. The strain factor has been computed by finite differences using data points for $\epsilon= \pm 0.4 \%$.

\begin{tabular}{lrrrrrr}
\hline$\Delta / J(0)$ & $\mu_{b}^{0}$ & $\mu_{c}^{0}$ & $g_{b b}$ & $g_{c b}$ & $g_{c c}$ & $g_{b c}$ \\
\hline 0 & 24.4 & 9.5 & 4.1 & 3.8 & -12.7 & -12.1 \\
0.5 & 13.0 & 5.1 & -0.1 & 0.1 & -14.8 & -14.0 \\
0.8 & 5.1 & 2.1 & -5.8 & -5.1 & -17.8 & -17.3 \\
\hline
\end{tabular}

Table 3 shows the strain factor tensor $g_{i j}=\frac{\partial\left(\Delta \mu_{i} / \mu_{0 i}\right)}{\partial \epsilon_{j}}$, quantifying the calculated anisotropic electro-mechanical response of the rubrene crystal as a function of the amount of extrinsic disorder. We observe that the magnitude of the mobility variations is larger for strain along 

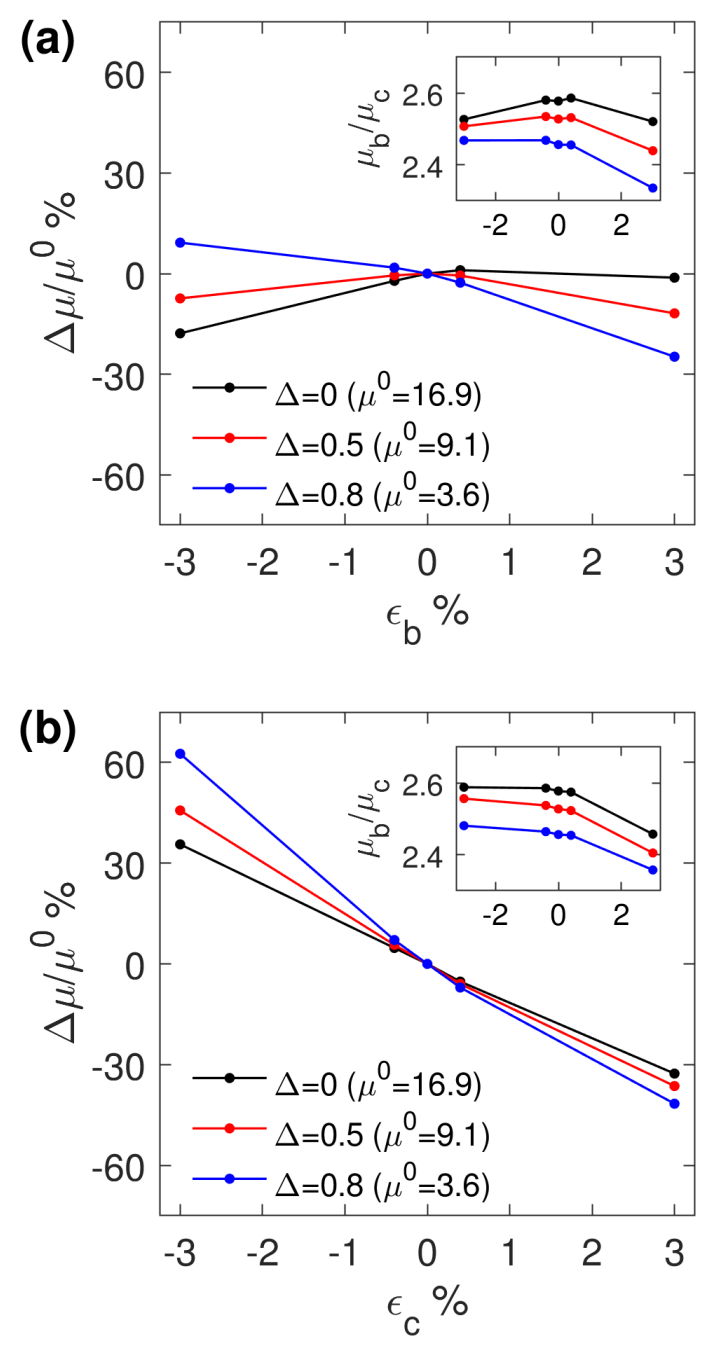

Figure 7: Relative mobility variations as a function of strain applied along (a) $b$ and (b) $c$ axis. Main panels refer to the mean mobility $\mu=\left(\mu_{b}+\mu_{c}\right) / 2$, insets show the $\mu_{b} / \mu_{c}$ ratio quantifying the anisotropy in the $b c$ plane. The curves for different values of the extrinsic disorder $\Delta$ (in units of $J(\epsilon=0)$, see legend), show that this factor favors mobility enhancements upon compression. The average $b c$-plane mobility (annotated in $\mathrm{cm}^{2} \mathrm{~V}^{-1} \mathrm{~s}^{-1}$ in the legend) degrades upon introducing extrinsic disorder. 
$c$ than along $b$, while, for a given strain direction, similar mobility variations are observed along the two axes. In fact, the ratio $\mu_{b} / \mu_{c}$ is about 2.5 and weakly depends on strain and on the extrinsic disorder $\Delta$, as shown in the insets of Figure 7. Indeed, within transient localization theory, the anisotropy in the mobility is approximately given by the anisotropy in the effective masses, ${ }^{18}$ which is minimally altered by strain or disorder.

The effect of strain on the mobility can be understood in terms of the combined effect of the variations in transfer integrals and in the energetic disorder. Within the transient localization theory, $\mu$ varies proportionally to $(J / \sigma)^{\alpha}$, where $\alpha \gtrsim 2$ and $\sigma$ quantifies the total energetic disorder due to local and nonlocal coupling. For one-dimensional systems, it can be shown that $\sigma^{2}=\sigma_{B}^{2}+2 \sigma_{C}^{2}+\left(\sigma_{H}^{2}+\Delta^{2}\right) / 4$. Numerical evidence suggests that the same formula holds to a good approximation also for generic two-dimensional band structures. ${ }^{56}$

Compressive strain applied along $c$ enhances the magnitude of transfer integrals without significantly affecting the total energetic disorder, hence resulting in an increase in $(J / \sigma)$, and therefore in mobility. On the other hand, the lattice compression along $b$ increases $\sigma_{B}$, whose relative variations are larger in magnitude than those in $J$. This reduces the ratio $(J / \sigma)$, resulting in a positive sign of the strain factor components $g_{i b}(i=b, c)$ for $\Delta=0$. Strain independent contributions to $\sigma$, such as the extrinsic disorder $\Delta$, are therefore crucial as they reduce the relative variations in $\sigma$, up to inverting the trend and reversing the sign of $g_{i b}$. Eventually, when extrinsic disorder is dominant, the variations in the mobility are solely governed by the changes in the magnitude of the transfer integrals.

\section{Discussion and conclusions}

In this paper, we have presented a theoretical analysis of the strain dependence of the mobility of the rubrene crystal combining first principles calculations and charge carrier mobility simulations. DFT calculations allowed for an accurate evaluation of intermolecular transfer integrals and energetic disorder as a function of the applied strain. Charge transport 
simulations based on the DFT inputs showed that the mobility response is larger when the strain is applied along the crystal axis $c$ with respect to $b$. In the former case, compression always results in an enhancement of $\mu$, while when strain is along $b$ it can, in principle, both increase or reduce $\mu$.

Our analysis highlights also the importance of the extrinsic energetic disorder, $\Delta$, which enhances the electro-mechanical response of the crystal. This result suggests that factors such as the purity of crystalline samples or the choice of the gate dielectric might be crucial for the electro-mechanical response of a given system, possibly rationalizing the large variability in experimental data. Indeed, for large $\Delta$ the trends of mobility with strain are mostly governed by the increase in the transfer integrals upon compression, rather than by the variations of intrinsic energetic disorder, which are found here to be modest in rubrene. This rationalizes the satisfactory explanation of the trends of mobility with strain that has been advanced by solely looking at the variations of transfer integrals. ${ }^{9,54}$

Although a precise quantification of the extrinsic disorder is difficult both in simulations and in experiments, useful indications can be obtained from the measured mobility values. The mobility of rubrene single crystals for which strain effects have been reported varies between 1 and $8 \mathrm{~cm}^{2} \mathrm{~V}^{-1} \mathrm{~s}^{-1} \cdot{ }^{6,8,9}$ These values lag far behind the highest mobilities reported for top-performing air-gap transistors, showing $\mu$ values up to $24 \mathrm{~cm}^{2} \mathrm{~V}^{-1} \mathrm{~s}^{-1}$, closely comparing to our results for extrinsic disorder-free rubrene. This observation suggests that extrinsic phenomena occur to substantially limit charge transport in the samples and devices for which the electro-mechanical response has been measured.

The strain factor $g$ we obtain for $\Delta / J_{0}=0.8$ corresponds to a $\sim 18 \%$ mobility increase for $1 \%$ compression along $c$ and a three times weaker response for strain along $b$. These values are in agreement with the $g$ factors measured by Matta et al. under tensile strain, ${ }^{9}$ both in terms of magnitude and anisotropy of the response. Our values are also comparable to those by Wang et al. $(g \sim 20),{ }^{8}$ while Reyes et al., using a peculiar experimental setup in which strain is induced exploiting the wrinkling of an elastomeric substrate, reported responses one 
order of magnitude larger. ${ }^{6}$

In summary, we have developed a theoretical and computational approach for the investigation of the mobility-strain relationship in crystalline molecular semiconductors that takes into account several factors, including dynamic energetic disorder due to lattice vibrations. We have rationalized the enhancement of the mobility with lattice compression in rubrene, mostly due by the increase in magnitude of intermolecular charge transfer integrals, with a possible contribution from extrinsic effects. In virtue of the specificity of the different classes of molecular crystals, we cannot exclude that other systems would present qualitatively different behaviors. The protocol proposed herein provides a robust framework for the investigation of the electro-mechanical response in different molecular crystals.

\section{Acknowledgement}

The authors thank V. Podzorov, L. Muccioli, S. M. Gali, D. Beljonne, A. Girlando, H. Sirringhaus and G. Schweicher for stimulating discussions and results sharing. Computational resources provided by the French GENCI/CEA-TGCC are acknowledged. MTR thanks the University of Vermont for support. S.F. acknowledges support by DFG (Grant No. DR228/48-1).

\section{References}

(1) Someya, T.; Sekitani, T.; Iba, S.; Kato, Y.; Kawaguchi, H.; Sakurai, T. A large-area, flexible pressure sensor matrix with organic field-effect transistors for artificial skin applications. Proc. Natl. Acad. Sci. USA 2004, 101, 9966-9970.

(2) Briseno, A.; Tseng, R.; Ling, M.-M.; Falcao, E.; Yang, Y.; Wudl, F.; Bao, Z. Highperformance organic single-crystal transistors on flexible substrates. Adv. Mater. 2006, 18, 2320-2324. 
(3) Gelinck, G.; Heremans, P.; Nomoto, K.; Anthopoulos, T. D. Organic transistors in optical displays and microelectronic applications. Adv. Mater. 2010, 22, 3778-3798.

(4) Yao, Y.; Dong, H.; Hu, W. Charge transport in organic and polymeric semiconductors for flexible and stretchable devices. Adv. Mater. 2016, 28, 4513-4523.

(5) Qian, Y.; Zhang, X.; Xie, L.; Qi, D.; Chandran, B. K.; Chen, X.; Huang, W. Stretchable organic semiconductor devices. Adv. Mater. 2016, 28, 9243-9265.

(6) Reyes-Martinez, M. A.; Crosby, A. J.; Briseno, A. L. Rubrene crystal field-effect mobility modulation via conducting channel wrinkling. Nat. Commun. 2015, 6, 6948.

(7) Kubo, T.; Häusermann, R.; Tsurumi, J.; Soeda, J.; Okada, Y.; Yamashita, Y.; Akamatsu, N.; Shishido, A.; Mitsui, C.; Okamoto, T. et al. Suppressing molecular vibrations in organic semiconductors by inducing strain. Nat. Commun. 2016, 7, 11156.

(8) Wang, H.; Deng, L.; Tang, Q.; Tong, Y.; Liu, Y. Flexible organic single-crystal fieldeffect transistor for ultra-sensitivity strain sensing. IEEE Electr. Device Lett. 2017, 38, 1598.

(9) Matta, M.; Pereira, M. J.; Gali, S. M.; Thuau, D.; Olivier, Y.; Briseno, A.; Dufour, I.; Ayela, C.; Wantz, G.; Muccioli, L. Unusual electromechanical response in rubrene single crystals. Mater. Horiz. 2018, 5, 41-50.

(10) Morf, T.; Mathis, T.; Batlogg, B. Unusual anisotropic response of the charge carrier mobility to uniaxial mechanical strain in rubrene crystals. arXiv:cond-mat.mtrl-sci 2016, 1606.06875 .

(11) Wu, Y.; Chew, A. R.; Rojas, G. A.; Sini, G.; Haugstad, G.; Belianinov, A.; Kalinin, S. V.; Li, H.; Risko, C.; Brédas, J.-L. et al. Strain effects on the work function of an organic semiconductor. Nat. Commun. 2016, 7, 10270. 
(12) Troisi, A.; Orlandi, G. Charge-transport regime of crystalline organic semiconductors: diffusion limited by thermal off-diagonal electronic disorder. Phys. Rev. Lett. 2006, 96, 086601.

(13) Ciuchi, S.; Fratini, S.; Mayou, D. Transient localization in crystalline organic semiconductors. Phys. Rev. B 2011, 83, 081202.

(14) Eggeman, A. S.; Illig, S.; Troisi, A.; Sirringhaus, H.; Midgley, P. A. Measurement of molecular motion in organic semiconductors by thermal diffuse electron scattering. Nat. Mater. 2013, 12, 1045.

(15) Illig, S.; Eggeman, A. S.; Troisi, A.; Jiang, L.; Warwick, C.; Nikolka, M.; Schweicher, G.; Yeates, S. G.; Henri Geerts, Y.; Anthony, J. E. et al. Reducing dynamic disorder in small-molecule organic semiconductors by suppressing large-amplitude thermal motions. Nat. Commun. 2016, 7, 10736.

(16) Wang, L.; Prezhdo, O. V.; Beljonne, D. Mixed quantum-classical dynamics for charge transport in organics. Phys. Chem. Chem. Phys. 2015, 17, 12395-12406.

(17) Fratini, S.; Mayou, D.; Ciuchi, S. The transient localization scenario for charge transport in crystalline organic materials. Adv. Funct. Mater. 2016, 26, 2292-2315.

(18) Fratini, S.; Ciuchi, S.; Mayou, D.; de Laissardière, G. T.; Troisi, A. A map of highmobility molecular semiconductors. Nat. Mater. 2017, 16, 998.

(19) Troisi, A. Prediction of the absolute charge mobility of molecular semiconductors: the case of rubrene. Adv. Mater. 2007, 19, 2000-2004.

(20) da Silva Filho, D.; Kim, E.-G.; Brédas, J.-L. Transport properties in the rubrene crystal: electronic coupling and vibrational reorganization energy. Adv. Mater. 2005, 17, 10721076. 
(21) Sundar, V. C.; Zaumseil, J.; Podzorov, V.; Menard, E.; Willett, R. L.; Someya, T.; Gershenson, M. E.; Rogers, J. A. Elastomeric transistor stamps: reversible probing of charge transport in organic crystals. Science 2004, 303, 1644-1646.

(22) Jurchescu, O. D.; Meetsma, A.; Palstra, T. T. M. Low-temperature structure of rubrene single crystals grown by vapor transport. Acta Crystallogr. B 2006, 62, 330-334.

(23) Wang, L. J.; Peng, Q.; Li, Q. K.; Shuai, Z. Roles of inter- and intramolecular vibrations and band-hopping crossover in the charge transport in naphthalene crystal. J. Chem. Phys. 2007, 127, 044506.

(24) Coropceanu, V.; Sánchez-Carrera, R. S.; Paramonov, P.; Day, G. M.; Brédas, J.-L. Interaction of charge carriers with lattice vibrations in organic molecular semiconductors: naphthalene as a case study. J. Phys. Chem. C 2009, 113, 4679-4686.

(25) Girlando, A.; Grisanti, L.; Masino, M.; Bilotti, I.; Brillante, A.; Della Valle, R. G.; Venuti, E. Peierls and holstein carrier-phonon coupling in crystalline rubrene. Phys. Rev. B 2010, 82, 035208.

(26) Sánchez-Carrera, R. S.; Paramonov, P.; Day, G. M.; Coropceanu, V.; Brédas, J.-L. Interaction of charge carriers with lattice vibrations in oligoacene crystals from naphthalene to pentacene. J. Am. Chem. Soc. 2010, 132, 14437.

(27) Girlando, A.; Grisanti, L.; Masino, M.; Brillante, A.; Della Valle, R. G.; Venuti, E. Interaction of charge carriers with lattice and molecular phonons in crystalline pentacene. J. Chem. Phys. 2011, 135, 084701.

(28) Perdew, J.; Burke, K.; Ernzerhof, M. Generalized gradient approximation made simple. Phys. Rev. Lett. 1996, 77, 3865-3868.

(29) Grimme, S.; Antony, J.; Ehrlich, S.; Krieg, H. A consistent and accurate ab initio 
parametrization of density functional dispersion correction (DFT-D) for the 94 elements H-pu. J. Chem. Phys. 2010, 132, 154104.

(30) Grimme, S.; Ehrlich, S.; Goerigk, L. Effect of the damping function in dispersion corrected density functional theory. J. Comput. Chem. 2011, 32, 1456-1465.

(31) Brandenburg, J. G.; Grimme, S. In Dispersion Corrected Hartree-fock and Density Functional Theory for Organic Crystal Structure Prediction.; Atahan-Evrenk, S., Aspuru-Guzik, A., Eds.; Prediction and Calculation of Crystal Structures. Topics in Current Chemistry; Springer International Publishing, 2014; Vol. 345.

(32) Bedoya-Martínez, N.; Giunchi, A.; Salzillo, T.; Venuti, E.; Della Valle, R.; Zojer, E. Toward a reliable description of the lattice vibrations in organic molecular crystals: the impact of van der waals interactions. J. Chem. Theory Comput. 2018, 14, 4380-4390.

(33) Dovesi, R.; Erba, A.; Orlando, R.; Zicovich-Wilson, C. M.; Civalleri, B.; Maschio, L.; Rérat, M.; Casassa, S.; Baima, J.; Salustro, S. et al. Quantum-mechanical condensed matter simulations with crystal. Wiley Interdiscip. Rev. Comput. Mol. Sci. 2018, 110, e1360.

(34) Pascale, F.; Zicovich-Wilson, C. M.; Gejo, F. L.; Civalleri, B.; Orlando, R.; Dovesi, R. The calculation of the vibrational frequencies of crystalline compounds and its implementation in the crystal code. J. Comput. Chem. 2004, 25, 888-897.

(35) Zicovich-Wilson, C. M.; Pascale, F.; Roetti, C.; Saunders, V. R.; Orlando, R.; Dovesi, R. Calculation of the vibration frequencies of alpha-quartz: the effect of hamiltonian and basis set. J. Comput. Chem. 2004, 25, 1873-1881.

(36) Dovesi, R.; Kirtman, B.; Maschio, L.; Maul, J.; Pascale, F.; Rérat, M. Calculation of the infrared intensity of crystalline systems. A comparison of three strategies based on berry phase, wannier function, and coupled-perturbed kohn-Sham methods. J. Phys. Chem. C 2018, acs.jpcc.8b08902. 
(37) Natkaniec, I.; Bokhenkov, E. L.; Dorner, B.; Kalus, J.; Mackenzie, G. A.; Pawley, G. S.; Schmelzer, U.; Sheka, E. F. Phonon dispersion in d8-naphthalene crystal at 6k. Journal of Physics C: Solid State Physics 1980, 13, 4265-4283.

(38) Tu, Z.; Yi, Y.; Coropceanu, V.; Brédas, J.-L. Impact of phonon dispersion on nonlocal electron-Phonon couplings in organic semiconductors: the naphthalene crystal as a case study. J. Phys. Chem. C 2017, 122, 44-49.

(39) Lee, N.-E.; Zhou, J.-J.; Agapito, L. A.; Bernardi, M. Charge transport in organic molecular semiconductors from first principles: the bandlike hole mobility in a naphthalene crystal. Phys. Rev. B 2018, 97, 115203.

(40) Xie, X.; Santana-Bonilla, A.; Troisi, A. Nonlocal electron-Phonon coupling in prototypical molecular semiconductors from first principles. J. Chem. Theory Comput 2018, 14, $3752-3762$.

(41) Yi, Y.; Coropceanu, V.; Brédas, J.-L. Nonlocal electron-phonon coupling in the pentacene crystal: beyond the -point approximation. J. Chem. Phys. 2012, 137, 164303.

(42) Tu, Z.; Yi, Y.; Coropceanu, V.; Brédas, J.-L. Impact of phonon dispersion on nonlocal electron-Phonon couplings in organic semiconductors: the naphthalene crystal as a case study. J. Phys. Chem. C 2018, 122, 44-49.

(43) Harrelson, T. F.; Dantanarayana, V.; Xie, X.; Koshnick, C.; Nai, D.; Fair, R.; Nuñez, S. A.; Thomas, A. K.; Murrey, T. L.; Hickner, M. A. et al. Direct probe of the nuclear modes limiting charge mobility in molecular semiconductors. Mater. Horiz. 2019, 6, 182-191.

(44) Adamo, C.; Barone, V. Toward reliable density functional methods without adjustable parameters: the PBE0 model. J. Chem. Phys. 1999, 110, 6158-6170. 
(45) Neese, F. The ORCA program system. Wiley Interdiscip. Rev. Comput. Mol. Sci. 2011, 2, 73-78.

(46) Neese, F.; Wennmohs, F.; Hansen, A.; Becker, U. Efficient, approximate and parallel hartree-Fock and hybrid DFT calculations. A 'chain-of-spheres' algorithm for the hartree-Fock exchange. Chem. Phys. 2009, 356, 98-109.

(47) Valeev, E. F.; Coropceanu, V.; da Silva Filho, D. A.; Salman, S.; Brédas, J.-L. Effect of electronic polarization on charge-transport parameters in molecular organic semiconductors. J. Am. Chem. Soc. 2006, 128, 9882-9886.

(48) Ciuchi, S.; Fratini, S. Electronic transport and quantum localization effects in organic semiconductors. Phys. Rev. B 2012, 86 .

(49) Nematiaram, T.; Ciuchi, S.; Xie, X.; Fratini, S.; Troisi, A. Practical computation of the charge mobility in molecular semiconductors using transient localization theory. $J$. Phys. Chem. C 2019, 123, 6989-6997.

(50) https://github.com/CiuK1469/TransLoc (accessed Feb 28, 2019).

(51) The average over disorder realizations can be performed in different ways. For dynamically thermalized disorder an annealed averaging procedure is in order, i.e. in Eq. 7 the numerator and the denominator $Z$ should be "averaged" separately. On the other hand, a quenched average, in which the whole Eq. 7 is averaged, is appropriate in the case of static disorder. give the same results. average scheme, as they proved to be much more stable and to quickly converge with the number of disorder realizations. We have checked that in absence of extrinsic disorder $(\Delta=0.0)$ the two methods give the same results, therefore in all cases we adopt the quenched average scheme to properly account for any source of disorder.

(52) Nan, G.; Yang, X.; Wang, L.; Shuai, Z.; Zhao, Y. Nuclear tunneling effects of charge transport in rubrene, tetracene, and pentacene. Phys. Rev. B 2009, 79, 115203. 
(53) Ordejón, P.; Boskovic, D.; Panhans, M.; Ortmann, F. Ab initio study of electron-phonon coupling in rubrene. Phys. Rev. B 2017, 96.

(54) Gali, S. M.; Quarti, C.; Olivier, Y.; Cornil, J.; Truflandier, L.; Castet, F.; Muccioli, L.; Beljonne, D. Impact of structural anisotropy on electro-mechanical response in crystalline organic semiconductors. J. Mater. Chem. C 2019, 7, 4382-4391.

(55) Ren, X.; Bruzek, M. J.; Hanifi, D. A.; Schulzetenberg, A.; Wu, Y.; Kim, C.-H.; Zhang, Z.; Johns, J. E.; Salleo, A.; Fratini, S. et al. Negative isotope effect on fieldeffect hole transport in fully substituted ${ }^{13}$ C-rubrene. Adv. Electron. Mater. 2017, 3, 1700018.

(56) Fratini, S.; Ciuchi, S. From transient localization to band transport: on quantum corrections and lack thereof. arXiv:cond-mat.mtrl-sci 2019, 1903.12603v2. 


\section{Author biography}

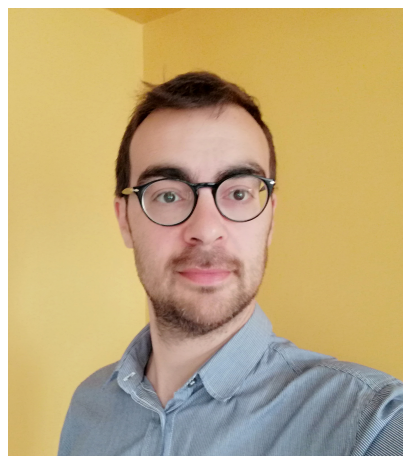

Gabriele D'Avino received his Ph.D. in Materials Science at the University of Parma in 2010. After a postdoctoral research position at the University of Bologna and two Marie Curie fellowships at the University of Liege and at the University of Mons, he is currently CNRS associate researcher at the Institut Néel in Grenoble. His research work focuses on the theoretical modeling of organic functional materials for advanced applications in electronics and energy generation, synergistically combining quantum and classical techniques in a multiscale fashion. The goal of his research is the establishment of the relationships between structure and (electronic, optical, vibrational, dielectric) properties in complex molecular systems with special emphasis on environmental and cooperative phenomena in the solid state. 


\section{Graphical TOC Entry}

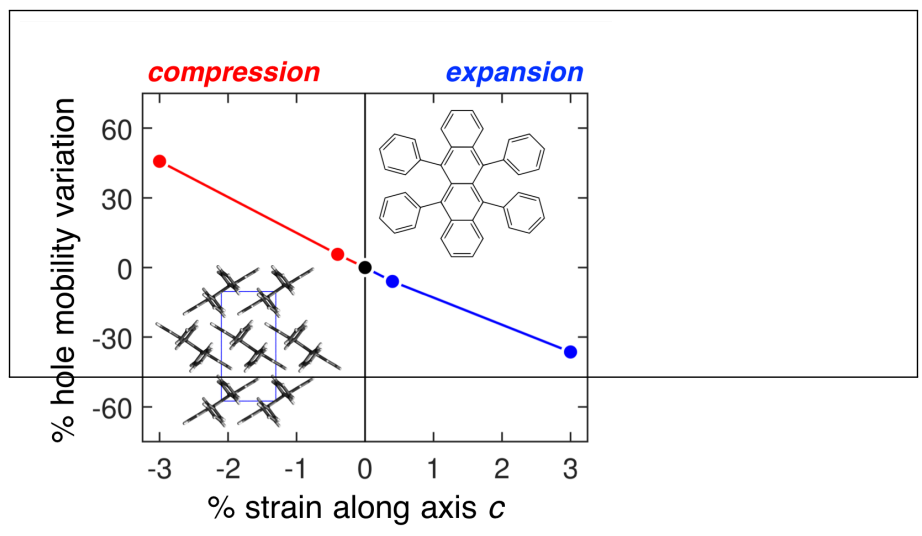

